\title{
Evaluation of Input Parameterization for Batch Process Optimization
}

\author{
C. Welz, B. Srinivasan, A. Marchetti and D. Bonvin ${ }^{\dagger}$ \\ Laboratoire d'Automatique \\ École Polytechnique Fédérale de Lausanne \\ CH-1015 Lausanne, Switzerland
}

\author{
N.L. Ricker \\ University of Washington \\ Seattle, WA 98195-1750, USA
}

\begin{abstract}
For the optimization of dynamic systems, it is customary to use measurements to combat the effect of uncertainty. In this context, an approach that consists of tracking the necessary conditions of optimality is gaining in popularity. The approach relies strongly on the ability to formulate an appropriate solution model, i.e. an approximate parameterization of the optimal inputs with a precise link to the necessary conditions of optimality. Hence, the need to be able to assess the capability of a solution model to optimize an uncertain process. This paper introduces a loss function that can be used to verify the conjecture that the solution model derived from a simplified process model can be applied to a more rigorous process model with negligible loss in performance. This conjecture is tested in simulation via the dynamic optimization of a batch distillation column.
\end{abstract}

Keywords: Dynamic optimization, Measurement-based optimization, Implicit optimization, NCO tracking, Batch distillation.

\section{Introduction}

A frequent objective in batch process operation is the maximization of product yield at final time while satisfying path and terminal constraints. In the presence of uncertainty (model mismatch and/or process disturbances), the constraints are typically met by applying a conservative policy that, unfortunately, can be far from optimal. For process improvement and thus reduction of this conservatism, it is necessary to use measurements. This can be accomplished via model refinement and re-optimization (explicit optimization) or by updating the inputs directly (implicit optimization). ${ }^{1}$ This paper considers a technique of the latter class, i.e. optimization via tracking of the necessary conditions of optimality ( $\mathrm{NCO}$ ).

NCO tracking treats the optimization problem as a feedback control problem, with the attendant advantages of sensitivity reduction and disturbance rejection. ${ }^{2}$ Since the solution of a

\footnotetext{
${ }^{*}$ Current address: Dept of Chemical Engineering, Ecole Polytechnique Montreal, Montreal, Canada H3C 3A7

${ }^{\dagger}$ Contact person: dominique.bonvin@epfl.ch
} 
dynamic optimization problem is typically discontinuous and consists of various intervals, the $\mathrm{NCO}$ include several parts that correspond to meeting the active constraints and zeroing certain sensitivities, both during the run and at final time. ${ }^{3}$ Some of these parts (conditions) can be enforced on-line, while the others need several successive runs to be met.

The NCO-tracking approach relies on the concept of solution model, which is a description of the input profiles from an optimality viewpoint. The solution model relates the various adjustable elements of the optimal inputs to the $\mathrm{NCO} .{ }^{1}$ It is based on the input trajectories obtained via numerical optimization of a nominal process model. Its construction involves input dissection, input parameterization and the generation of links between the free elements of the inputs and the different parts of the NCO: Input dissection consists of decomposing the input profiles into various intervals and identifying those elements that vary with uncertainty; input parameterization is done so as to ease adaptation towards optimality. Finally, the input arcs and parameters that need to be adapted are linked to the appropriate NCO.

An important issue in NCO tracking is the evaluation of the solution model's accuracy, i.e. its flexibility for approximating the optimal policy. In general, increasing the number of input parameters increases the accuracy and thus improves the objective function, but it can also make parameter adaptation more difficult. ${ }^{4,5}$ Also, different types of parameterization may lead to different levels of accuracy. An automated method for determining the structure of the optimal solution, i.e. the various arcs and the switching times between them, using multi-stage numerical optimization has been proposed recently. ${ }^{6}$ This approach helps keep both the approximation error and the number of parameters small. The important problem of verifying whether the set of active constraints is invariant with respect to uncertainty has also been addressed recently. ${ }^{7}$ Evaluating the accuracy of a given input parameterization has been an important topic in the numerical optimization literature, for which measures that use the adjoint variables have been proposed. ${ }^{8}$ Yet, it has been suggested to use these measures as qualitative rather than quantitative indicators. In the same spirit, a simple loss function that expresses the loss with respect to truly optimal operation is proposed here.

A related issue is the solution model's applicability to the real process. The solution model is typically obtained through numerical optimization of a simplified process model. Hence, the important question: Does the solution model also hold for a more rigorous process model and, hopefully, also for the real process? In this paper, this issue is tackled by comparing the value of the loss function for both a tendency model and a much more detailed model of the same process.

The fact that a solution model obtained from a simplified process model can be equally applicable to more rigorous process models will be illustrated in simulation via the optimization of a binary batch distillation column. The objective will be to determine the reflux ratio policy that maximizes the final distillate quantity while meeting a purity constraint on the distillate composition for a given final time. Numerous studies have considered the dynamic optimization of batch distillation columns. For binary systems, three different operating strategies can be distinguished: ${ }^{9}$ a) constant reflux ratio, ${ }^{10}$ b) constant distillate composition, ${ }^{11}$ and c) optimal operation with time-varying reflux ratio. ${ }^{11-16}$ The latter strategy is considered in this study and includes the two former strategies as special cases. Note that the same methodology can also be applied to reactive batch distillation columns. ${ }^{5,17-19}$

The paper is organized as follows. The concept of dynamic optimization via NCO tracking is introduced and illustrated on the example of a binary batch distillation column in the next section. Then, we present a loss function that can be used for evaluating both the robustness of alternative solution models and the applicability of a solution model to a real plant. Finally, 
the utilization of the loss function is shown for the batch distillation example.

\section{Dynamic Optimization via NCO Tracking}

\section{Problem Formulation}

The following terminal-cost optimization of dynamic processes with free terminal time and path and terminal constraints is considered:

$$
\begin{array}{rll}
\min _{u(t), t_{f}} & J=\phi\left(x\left(t_{f}\right)\right) \\
\text { s.t. } & \dot{x}=F(x, u), & x(0)=x_{0} \\
& S(x, u) \leq 0, \quad T\left(x\left(t_{f}\right)\right) \leq 0
\end{array}
$$

where $\phi$ is the scalar cost function, $x$ the state vector with known initial conditions $x_{0}, u$ the input vector, and $t_{f}$ the final time. $F$ are the functions describing the system dynamics, $S \leq 0$ the path constraints, and $T \leq 0$ the terminal constraints.

In general, the solution of problem (1) is discontinuous and consists of a sequence of arcs or intervals. ${ }^{3}$ Within each interval, the inputs are continuous and differentiable. The time instants at which the inputs switch from one arc to another are called switching times. Two different types of arcs can be distinguished: an input is either determined by an active path constraint (constraint-seeking arc) or is inside the feasible region (sensitivity-seeking arc).

\section{Illustrative Example}

A binary batch distillation column is considered in order to illustrate the NCO concepts throughout this paper.

- Process model: A conventional binary batch distillation configuration is assumed, where the mixture of cyclohexane - n-heptane to be separated is charged initially into the reboiler, and the distillate is withdrawn from the condenser.

- Objective: Maximize the distillate quantity for a given final time $t_{f}$.

- Manipulated input: Internal reflux ratio $r(t)$.

- Path constraints: Bounds on the input, $0 \leq r(t) \leq 1$.

- Terminal constraint: Minimal distillate purity at final time, $x_{D}\left(t_{f}\right) \geq x_{D \text {,des }}$.

Model equations: Ideal vapor-liquid equilibrium of the binary system is assumed. The model comprises dynamic equations for the component holdup in the reboiler and in the condenser. Stage holdup is assumed to be negligible, and the relationship between the reboiler and condenser compositions is described by a shortcut method. ${ }^{20}$ The differential-algebraic equation system is written as:

$$
\begin{array}{ll}
\frac{d H_{1,1}}{d t}=V\left[r x_{p+1}-y_{p}\right] & H_{1,1}(0)=H_{1,1}^{0} \\
\frac{d H_{1,2}}{d t}=V\left[r\left(1-x_{p+1}\right)-\left(1-y_{p}\right)\right] & H_{1,2}(0)=H_{1,2}^{0}
\end{array}
$$




$$
\begin{array}{rlr}
\frac{d H_{p+1,1}}{d t} & =V\left(y_{p}-x_{p+1}\right) & H_{p+1,1}(0)=H_{p+1,1}^{0} \\
H_{1} & =H_{1,1}+H_{1,2} \\
H_{p+1} & =H_{p+1,1}+H_{p+1,2} \\
x_{1} & =\frac{H_{1,1}}{H_{1}} \\
x_{p+1} & =\frac{H_{p+1,1}}{H_{p+1}} \\
\frac{x_{1}}{\left(1-y_{p}\right)} & =\frac{\alpha}{\left(1-x_{1}\right)}\left(\frac{\alpha}{\sqrt{1+\frac{1-r}{r x_{1}}}}\right)^{\beta p} \\
x_{D}(t) & =\frac{\sum_{i=1}^{p+1}\left(H_{i, 1}(t)-H_{i, 1}(0)\right)}{\sum_{i=1}^{p+1}\left(H_{i}(t)-H_{i}(0)\right)}
\end{array}
$$

Variables and parameters: $H_{i, c}$ : holdup of component $c$ on stage $i, c=\{1,2\}$ for a binary mixture, $i=1$ : reboiler, $i=p+1$ : condenser, $V$ : boilup rate, $x_{i}$ : liquid composition on stage $i, y_{i}$ : vapor composition on stage $i, r$ : internal reflux ratio, $H_{i}$ : total holdup on stage $i, \alpha$ : relative volatility, $\beta$ : stage efficiency, $x_{D}$ : accumulated distillate composition.

\section{Optimization problem}

$$
\begin{aligned}
\max _{r(t)} J= & H_{1}(0)-H_{1}\left(t_{f}\right) \\
\text { s.t. } & \text { equations }(2)-(10) \\
& 0 \leq r(t) \leq 1 \\
& x_{D}\left(t_{f}\right) \geq x_{D, \text { des }}
\end{aligned}
$$

The model parameters and initial conditions are given in Table 1.

\begin{tabular}{cccccc}
\hline$H_{1,1}^{0}$ & 1.42 & $k m o l$ & $H_{1,2}^{0}$ & 1.42 & $k m o l$ \\
$H_{p+1,1}^{0}$ & 0.045 & $k m o l$ & $H_{p+1}$ & 0.09 & $k m o l$ \\
$p$ & 20 & & $t_{f}$ & 3 & $h$ \\
$\alpha$ & 1.7 & & $x_{D, \text { des }}$ & 0.95 & $\mathrm{kmol} / \mathrm{kmol}$ \\
$\beta$ & 0.7 & & $V$ & 3 & $\mathrm{kmol} / \mathrm{h}$ \\
\hline
\end{tabular}

Table 1: Model parameters and initial conditions.

Optimal solution: The optimal reflux ratio profile consists of 3 intervals (Figure 1): In Interval $a$, the reflux ratio is at its upper bound in order to accumulate the light component in the condenser. Then, in Interval b, distillate is withdrawn from the column at some intermediate reflux ratio that represents a compromise between quantity and quality. Finally, in Interval c, the reflux ratio is at its lower bound for emptying as quickly as possible the condenser that still contains distillate at high purity.

\section{Necessary Conditions of Optimality}

Define the following functions:

$$
H(t)=\lambda^{T} F(x, u)+\mu^{T} S(x, u) \quad \Phi\left(x\left(t_{f}\right)\right)=\phi\left(x\left(t_{f}\right)\right)+\nu^{T} T\left(x\left(t_{f}\right)\right)
$$




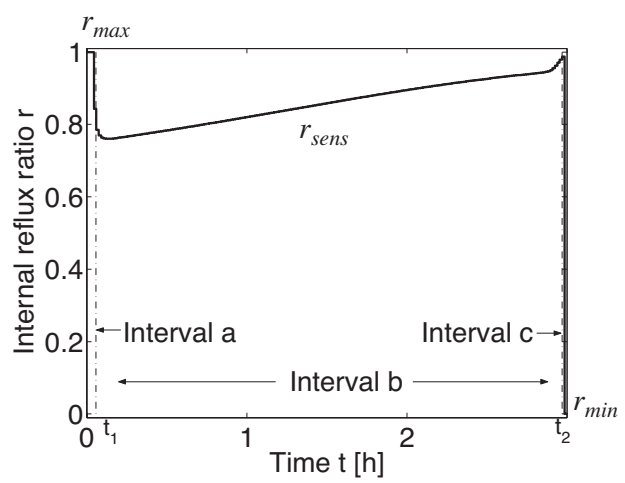

Figure 1: Optimal reflux ratio profile for illustrative example. The optimal profile is calculated via control vector iteration ${ }^{9,23}$ using 200 piecewise-constant elements.

$$
\Psi\left(x\left(t_{f}\right)\right)=\Phi\left(x\left(t_{f}\right)\right)+\int_{0}^{t_{f}} H(t) d t \quad \dot{\lambda}^{T}(t)=-\frac{\partial H}{\partial x}, \quad \lambda^{T}\left(t_{f}\right)=\frac{\partial \Phi}{\partial x\left(t_{f}\right)}
$$

$H(t)$ is the Hamiltonian function, $\Phi\left(x\left(t_{f}\right)\right)$ the augmented terminal cost, $\Psi\left(x\left(t_{f}\right)\right)$ the total terminal cost, $\lambda(t) \neq 0$ the $n$-dimensional vector of adjoint variables (Lagrange multipliers for the system equations), $\mu(t) \geq 0$ the $\zeta$-dimensional vector of Lagrange multipliers for the path constraints, and $\nu \geq 0$ the $\tau$-dimensional vector of Lagrange multipliers for the terminal constraints.

The NCO for Optimization problem (1) can be written as follows: ${ }^{3,21}$

\begin{tabular}{lll}
\hline & Path objectives & Terminal objectives \\
\hline Constraints & $\mu^{T} S(x, u, \rho)=0, \quad \mu \geq 0$ & $\nu^{T} T\left(x\left(t_{f}\right), \rho\right)=0, \quad \nu \geq 0$ \\
Sensitivities & $\frac{\partial H}{\partial u}=0$ & $\frac{\partial \Psi}{\partial \rho}=0$ \\
\hline
\end{tabular}

The NCO include both path and terminal objectives since there are conditions that have to be met during the operation, while others need to be satisfied only at final time. Also, optimality implies keeping certain constraints active and forcing certain sensitivities to zero. The Lagrange multipliers $\mu(t)$ and $\nu$ are zero when the corresponding constraints are $<0$ and nonzero otherwise so that $\mu^{T} S=0$ and $\nu^{T} T=0$ always when the constraints are satisfied (complementarity conditions). ${ }^{21}$

Since the inputs are typically discontinuous, it is helpful to treat the switching times as explicit decision variables. The input parameter vector that includes the switching times, the final time and the additional parameters resulting from parameterization of input arcs is denoted by $\pi$. The time functions that are left as infinite-dimensional variables constitute the vector of input arcs $\eta(t)$. The decision variables can be expressed as $\left\{u(t), t_{f}\right\}=\mathcal{U}\left(\eta\left[t_{s}, t_{s}^{\prime}\right), \pi\right)$, where the notation $\left[t_{s}, t_{s}^{\prime}\right)$ is used to indicate the interval for which a given arc $\eta_{i}(t)$ exists. With this notation, the sensitivity parts of the NCO in (14) can be written as:

$$
\frac{\partial H}{\partial \eta}\left[t_{s}, t_{s}^{\prime}\right)=0 \quad \frac{\partial \Psi}{\partial \pi}=0
$$

\section{NCO for Illustrative Example}

The NCO for the illustrative example are shown in Table 2. In the first interval $\left[0, t_{1}\right)$, the upper 
bound on the reflux ratio is active, $r\left[0, t_{1}\right)=1$, while in the last interval $\left[t_{2}, t_{f}\right)$ the lower bound is active, $r\left[t_{2}, t_{f}\right)=0$. Interval $b$ is determined by a path sensitivity condition. At final time, the terminal constraint $x_{D}\left(t_{f}\right)=x_{D, \text { des }}$ is active, which fixes the switching time $t_{1}$. The remaining input parameter, the switching time $t_{2}$, is sensitivity seeking and can be adjusted to improve the performance.

\begin{tabular}{ccc}
\hline & Path & Terminal \\
\hline Constraints & $r_{\text {max }}: r\left[0, t_{1}\right)=1$ & $t_{1}: x_{D}\left(t_{f}\right)=x_{D, \text { des }}$ \\
& $r_{\text {min }}: r\left[t_{2}, t_{f}\right)=0$ & \\
Sensitivities & $r_{\text {sens }}: \frac{\partial H}{\partial r}\left[t_{1}, t_{2}\right)=0$ & $t_{2}: \frac{\partial \Psi}{\partial t_{2}}=0$ \\
\hline
\end{tabular}

Table 2: NCO for the illustrative example.

\section{NCO Tracking}

NCO tracking aims at achieving optimality, also in the presence of perturbations, by treating the optimization problem as a control problem. NCO tracking enforces the four components of (14), some on-line and the others over successive batches: ${ }^{1,2}$

- Path constraints limit the values that the inputs or the states can take. Input bounds are straightforward to enforce by setting the inputs at their corresponding bounds. State constraints, which represent key safety and operational limitations that are assumed to be measurable, can be enforced by on-line feedback control. On the other hand, the evaluation of path sensitivities requires the use of a process model.

- Terminal constraints are typically kept active by measuring the constrained variables at final time and updating the inputs in the next run. Terminal sensitivities can also be met on a run-to-run basis by estimating them using either a process model or measurements of the terminal cost.

The assignment of time functions $\eta\left[t_{s}, t_{s}^{\prime}\right)$ and time-invariant input parameters $\pi$ to the different components of (14) constitutes the solution model. The generation of a solution model involves the following three tasks:

1. Determination of the switching structure of the optimal solution, i.e. the sequence and type of intervals present in the solution of (1). For this, numerical optimization of a nominal (tendency) process model is the method of choice. Arcs are typically detected by visual inspection, though an automated method for determining the switching structure has been proposed recently. ${ }^{6}$

Note that the structure detection step needs to uncover not only the structure of the optimal solution but also the amount of uncertainty for which this structure will still hold. Therefore, in addition to a nominal process model, this step requires good knowledge of the type and amount of uncertainty to be expected. 


\section{Structure for Illustrative Example}

The optimal input (Figure 1) can be written as:

$$
r(t)= \begin{cases}r_{\max } & \text { for } 0 \leq t<t_{1} \\ r_{\text {sens }}(t) & \text { for } t_{1} \leq t<t_{2} \\ r_{\min } & \text { for } t_{2} \leq t<t_{f}\end{cases}
$$

where $r_{\text {sens }}(t)$ is a sensitivity-seeking arc. In addition to input bounds that are active in the first and the last interval, the terminal constraint on distillate purity, $x_{D}\left(t_{f}\right)=x_{D \text {,des }}$, is active at final time.

2. Determination of the input fixed and free variables. The elements of the inputs that are not affected by uncertainty are considered as fixed in the solution model and can be applied in an open-loop fashion, e.g. an input variable at its bound in a given interval. The input elements affected by uncertainty constitute the free (decision) variables of the optimization problem. These include time functions (arcs) and time-invariant parameters (switching times and possibly the final time $t_{f}$ ). Furthermore, since it is easier to deal with scalar values than with time functions, certain input arcs can be parameterized using a small number of parameters, e.g. using a piecewise-polynomial representation.

\section{Fixed and Free Variables for Illustrative Example}

The sensitivity-seeking arc $r_{\text {sens }}\left[t_{1}, t_{2}\right)$ in Interval $b$ (Figure 1) can be described by a piecewise-linear profile with parameters $r_{1}$ at $t_{1}$ and $r_{2}$ at $t_{2}$. The optimal input can then be expressed as:

$$
r(t)= \begin{cases}1 & \text { for } \quad 0 \leq t<t_{1} \\ r_{1}+\left(t-t_{1}\right) \frac{r_{2}-r_{1}}{t_{2}-t_{1}} & \text { for } t_{1} \leq t<t_{2} \\ 0 & \text { for } t_{2} \leq t<t_{f}\end{cases}
$$

Let's assume that the relative volatility and the condenser holdup are uncertain parameters in the range $\alpha=\left[\begin{array}{ll}1.4 & 2\end{array}\right]$ and $H_{p+1}=\left[\begin{array}{ll}0 & 0.32\end{array}\right]$, respectively. The optimal input profiles for different values of the parametric uncertainty are shown in Figure 2. The structure of the optimal solution does not change with parametric uncertainty, i.e. the same three arcs $r_{\max } . r_{\text {sens }}$ and $r_{\min }$ are still present. On the other hand, the switching times between intervals as well as the reflux ratio parameters $r_{1}$ and $r_{2}$ in Interval $b$ change with uncertainty. Therefore, the parameters $\pi=\left[r_{1}, r_{2}, t_{1}, t_{2}\right]^{T}$ need to be adjusted and thus are selected as manipulated variables.

\section{Linking the decision variables to the various parts of the NCO.}

The fixed parts of the inputs are known and can be implemented directly without feedback. In contrast, the free parts (decision variables) need adjustment, and the $\mathrm{NCO}$ can be used for that purpose. The active path and terminal constraints determine certain arcs and parameters as will be shown next. The remaining decision variables are used to meet the path and terminal sensitivities. Through this assignment, a distinctive label can be tagged on the various input elements $\eta$ and $\pi$ as follows:

- The path constraint $(P C)$ variables $\eta_{P C}(t)$ and $\pi_{P C}$ can be adjusted by making the corresponding path constraints active.

- The terminal constraint (TC) variables $\eta_{T C}(t)$ and $\pi_{T C}$ can be adjusted by making the corresponding terminal constraints active. 

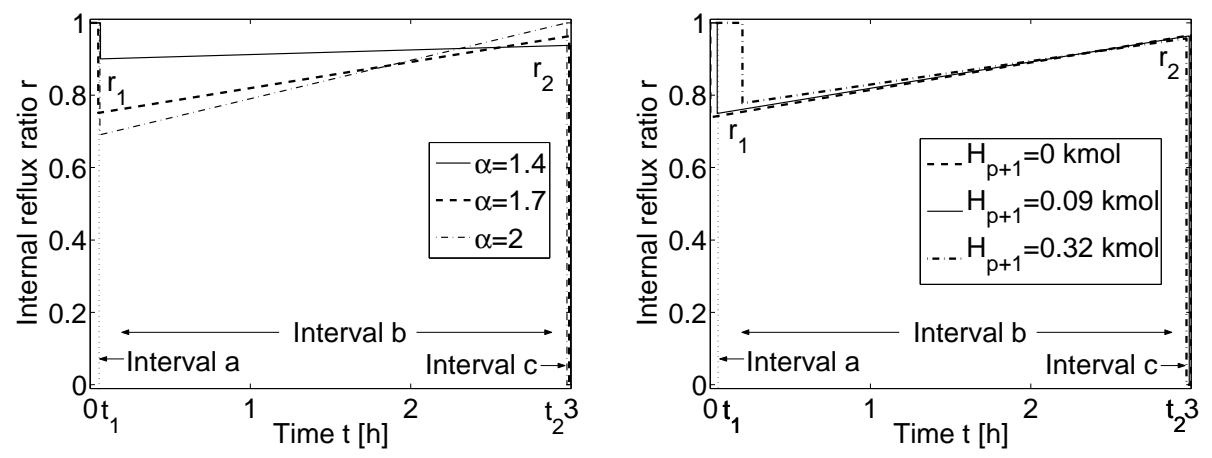

Figure 2: Optimal profiles for the nominal conditions but different values of the relative volatility $\alpha$ (left) and condenser holdup $H_{p+1}$ (right). The sensitivity-seeking arc is parameterized as a linear profile.

- The path sensitivity $(P S)$ variables $\eta_{P S}(t)$ need path sensitivity measurement or estimation for adaptation.

- The terminal sensitivity (TS) variables $\pi_{T S}$ need terminal sensitivity measurement or estimation for adaptation.

The update of the PC and TC variables are based on the measurement or estimation of path and terminal constraints, respectively. Similarly, the update of the PS and TS variables are based on the measurement or estimation of sensitivities. With $\bar{S}$ and $\bar{T}$ denoting the active state and terminal constraints, the decision variables of the optimal inputs can be expressed generically as:

$$
\begin{array}{ll}
\left\{\eta_{P C}\left[t_{s}, t_{s}^{\prime}\right), \pi_{P C}\right\}=\mathcal{P}_{c}(\bar{S}) & \left\{\eta_{T C}\left[t_{s}, t_{s}^{\prime}\right), \pi_{T C}\right\}=\mathcal{T}_{c}(\bar{T}) \\
\eta_{P S}\left[t_{s}, t_{s}^{\prime}\right)=\mathcal{P}_{s}\left(\frac{\partial H}{\partial \eta_{P S}}\right) & \pi_{T S}=\mathcal{T}_{s}\left(\frac{\partial \Psi}{\partial \pi_{T S}}\right)
\end{array}
$$

where $\mathcal{P}_{c}, \mathcal{T}_{c}, \mathcal{P}_{s}$ and $\mathcal{T}_{s}$ are appropriate operators/controllers for the path and terminal constraints and sensitivities, respectively. Equations (16)-(17) express the solution model in mathematical terms. They enforce a certain pairing between the decision variables and the NCO (constraints and sensitivities). As with any multi-loop control problem, this assignment requires designer judgment. Different pairings between the decision variables on the one hand and the NCO parts on the other will imply different adaptation strategies and thus different solution models.

An important assumption for this assignment to be effective is that the set of active constraints is correctly determined and does not vary with uncertainty. Fortunately, this restrictive assumption can often be relaxed by considering a super-structure for the constraints. ${ }^{1}$ 


\section{Solution Model for Illustrative Example}

The link between the decision variables of the input and the NCO is as follows:

\begin{tabular}{c|c|c|c} 
& \multicolumn{2}{|c|}{ Path } & \multicolumn{2}{c}{ Terminal } \\
\hline Constraints & $\eta_{1}(t): r\left[0, t_{1}\right)=1 \quad($ Interval $a)$ & \multicolumn{2}{|c}{$\pi_{1}=r_{1}: x_{D}\left(t_{f}\right)=x_{D, \text { des }}$} \\
& $\eta_{2}(t): r\left[t_{2}, t_{f}\right)=0 \quad($ Interval $c)$ & \multicolumn{2}{|c}{$\mid$} \\
\hline Sensitivities & - & $\pi_{2}:\left.\frac{\partial \Psi}{\partial \pi_{2}}\right|_{\bar{T}=0}=0$ with $\pi_{2}=\left[t_{1}, t_{2}, r_{2}\right]^{T}$
\end{tabular}

The reflux ratio $r$ is fixed at its upper and lower bounds in Intervals a and $c$, respectively. The reflux ratio parameter $r_{1}$ is adapted in order to meet the terminal constraint $x_{D}\left(t_{f}\right)=$ $x_{D, \text { des. }}$. The constrained cost sensitivity $\left.\frac{\partial \Psi}{\partial \pi_{2}}\right|_{\bar{T}=0}$ is evaluated under the terminal condition $x_{D}\left(t_{f}\right)=x_{D \text {,des }}$ and used for adapting the parameters $\pi_{2}$. Note that, upon parameterization of the sensitivity arc in Interval $b$, the path sensitivity condition $\frac{\partial H\left[t_{1}, t_{2}\right)}{\partial r}=0$ in Table 2 is replaced by two terminal sensitivity conditions of the type $\left.\frac{\partial \Psi}{\partial \pi_{2}}\right|_{\bar{T}=0}=0$, which are straightforward to evaluate at the end of each run. Hence, the adaptation of the parameter $r_{1}, t_{1}, t_{2}$ and $r_{2}$ will be done on a run-to-run basis so as to achieve both $x_{D}\left(t_{f}\right)=x_{D \text {,des }}$ and $\left.\frac{\partial \Psi}{\partial \pi_{2}}\right|_{\bar{T}=0}=0$. This solution model is referred to as Solution Model $A$ in the following.

The formulation of a solution model involves simplifications and approximations that help make the NCO-tracking problem more tractable and efficient. Simplifications can be introduced at various levels. For example, one can neglect arcs that contribute little to performance or hold an input constant during a period in which it would otherwise change only slightly. Also, as mentioned above, it is convenient to convert certain sensitivity-seeking arcs $\eta_{P S}$ to sensitivityseeking parameters $\pi_{T S}$ since the latter are easy to evaluate at run end. The sensitivity-seeking arcs can be approximated using piecewise-polynomial (e.g. piecewise-linear) or exponential functions. At this point, it is important to realize that the optimization problem (1) has been approximated by the decentralized control problem (18). Since process measurements are used for implementation, NCO tracking is not sensitive to model mismatch and, in addition, it is robust with respect to process disturbances.

\section{Alternative Solution Models for Illustrative Example}

The following two alternative solution models will also be considered.

- In Solution Model B, the last interval (and thus also $t_{2}$ ) is eliminated. There results an optimization problem with the input parameters $t_{1}, r_{1}$ and $r_{2}$, i.e. $n_{\pi}=3$.

- In Solution Model $C$, the reflux ratio is additionally kept constant in Interval $b\left(n_{\pi}=2\right.$, with the parameters $t_{1}$ and $r_{1}$ ).

For Solution Models $B$ and $C$, linking the input parameters $\pi$ to the NCO is similar to Solution Model $A$, the only difference being that $\pi_{2}$ has now two and one parameters, respectively.

\section{Solution Model Validation}

As described in the previous section, a solution model is a tool used to describe, possibly in an approximate way, the optimal inputs and allow their adaptation using measurements. 
There might be several solution model candidates for a given optimization problem. Hence, it is important to compare them and assess the quality of the approximation. For this, a loss function is introduced next.

\section{Loss Function}

Let $u^{*}$ be the true optimal input, and $u^{s}$ a candidate policy whose quality is to be determined. Also, let $J\left(u^{*}\right)$ and $J\left(u^{s}\right)$ be the corresponding cost functions evaluated on the real plant (or its representation in a simulation study).

A simple way of assessing the distance of the proposed solution to the true optimum is to define the loss function

$$
\Theta_{\text {loss }}=\frac{J\left(u^{s}\right)-J\left(u^{*}\right)}{\left|J\left(u^{s}\right)\right|}
$$

which normalizes the difference $J\left(u^{s}\right)-J\left(u^{*}\right)$ with respect to the obtained cost $\left|J\left(u^{s}\right)\right|$. The absolute value is introduced here to normalize with a positive number since the cost function can be negative, e.g. upon transforming a maximization problem into the minimization formulation (1). Normalization helps compare loss functions for different processes. The measure $\Theta_{\text {loss }}$ is positive or zero. Note that, for a maximization problem, one would use $J\left(u^{*}\right)-J\left(u^{s}\right)$ in the numerator of $\Theta_{\text {loss }}$.

This loss function requires knowledge of the true optimal cost $J\left(u^{*}\right)$, which is typically not available in practical applications. Even in a simulation study, $u^{*}$ is difficult to calculate since it necessitates an infinite-dimensional parameterization. However, in the absence of path constraints, a good approximation of the cost $J\left(u^{*}\right)$ - though not necessarily of the inputs $u^{*}-$ can be obtained by increasing the number of parameters and extrapolation. ${ }^{22}$ In the presence of path constraints, extrapolation will typically fail each time an additional constraint becomes active as the result of more flexibility in the inputs. Yet, when the set of active constraints does not change with additional input parameters, extrapolation can be made.

\section{Robustness of Solution Model}

The loss function $\Theta_{\text {loss }}$ can be used to evaluate the quality of approximation of a given solution model. This section investigates the robustness of the solution model by assessing whether the solution model that is derived from - and found appropriate for - a simple process model can be applied to different models of the same process with negligible loss in performance. It is important to realize that the robustness test is with respect to the solution model and not the optimal solution. In other words, though the optimal solution may vary significantly due to parametric and structural uncertainty between the various models, the link between the decision variables and the NCO that is used to achieve (near) optimality remains valid. The main conjecture addressed in this paper is presented next.

Conjecture : A solution model is insensitive to "reasonable variations" in the parameters or the structure of a plant model if the loss function $\Theta_{\text {loss }}$ remains close to zero.

The conjecture can be understood as follows. Consider a process for which the two models $\mathcal{M}_{1}$ and $\mathcal{M}_{2}$ are available. Let the solution model $\mathcal{S}$ be available and $\Theta_{\text {loss }}\left(\mathcal{S}, \mathcal{M}_{i}\right)$ represent the loss function involving $J\left(u^{s}\right)$ computed by $\mathrm{NCO}$ tracking using the solution model and $J\left(u^{*}\right)$ computed numerically from the process model $\mathcal{M}_{i}$. Suppose the solution model has been found 
appropriate for process model $\mathcal{M}_{1}$, i.e. $\Theta_{\text {loss }}\left(\mathcal{S}, \mathcal{M}_{1}\right) \simeq 0$. Then, if $\Theta_{\text {loss }}\left(\mathcal{S}, \mathcal{M}_{2}\right) \simeq 0$, the solution model $\mathcal{S}$ also holds for $\mathcal{M}_{2}$.

The conjecture says that the loss function $\Theta_{\text {loss }}$ (and not the cost function $J$ ) is insensitive to variations in the process model. In other words, if the solution model is adequate for optimizing correctly different models of the same process, $\Theta_{\text {loss }}$ remains nearly constant though $J$ may change significantly. This indicates that the sequence of arcs and the associated input parameters are judiciously chosen and the solution model applies equally well to all process models. Conversely, if the loss function varies significantly in response to parameter and/or structural variations, the corresponding solution model is not appropriate.

The conjecture can always be falsified by sufficiently large uncertainty. However, as will be shown on the illustrative example, the conjecture can indeed be verified for a reasonable range of parametric variations and rather large variations in the model structure.

\section{Solution Model for the Real Plant}

If the solution model has been found insensitive to "reasonable variations" in the parameters or the structure of a plant model, it is hoped that the same carries over to the real plant. This is particularly true if these reasonable variations around the nominal plant cover the unknown real plant.

The following procedure is then proposed for generating and validating a solution model capable of optimizing a real plant:

1. Use a simple nominal plant model, generate the corresponding solution model, perform NCO tracking with it and compute the loss function $\Theta_{\text {loss }}$ using the nominal model as simulated plant. This will validate some of the approximations introduced in the solution model and indicate whether the solution model has sufficient flexibility for optimizing the nominal plant model.

2. Assess the amount of model mismatch between the plant model and the real plant and bound it in terms of parametric variations for the nominal model, i.e. determine one or several worst-case scenarios using the nominal plant model structure.

3. Using the solution model of Step 1, perform NCO tracking on these worst-case plant models and compute the associated loss functions.

4. If all loss functions are close to zero, the solution model is valid for all worst-case scenarios and, hopefully, also for the real plant. Otherwise, it is necessary to iterate and try to obtain a more detailed nominal plant model with reduced plant/model mismatch.

\section{Validation of Solution Models for Illustrative Example}

\section{Loss Function for Tendency Process Model}

The optimization results for the tendency model that was presented as illustrative example are listed in Table 3. The optimal cost $J\left(u^{*}\right)$ is calculated via control vector iteration ${ }^{9}$ using 200 piecewise-constant elements (Figure 1). The costs $J\left(u^{s}\right)$ are obtained by NCO tracking with the solution models A, B and C. It is seen from $\Theta_{\text {loss }}$ that a parameterization with only 4 parameters 
(Solution Model A) only affects optimality by $0.2 \%$. Furthermore, the elimination of Interval c in Solution Model B barely affects the performance, while a constant reflux in Interval b (Solution Model C) causes noticeable deterioration in performance. Hence, Solution Models A and $\mathrm{B}$ are rather appropriate since the corresponding loss functions $\Theta_{\text {loss }}$ are close to 0.

Table 3: Cost $J$ and loss function $\Theta_{\text {loss }}$ for the tendency process model. The solution models A-C are generated from numerical optimization of the tendency process model.

\begin{tabular}{ccc}
\hline Strategy & $J[\mathrm{kmol}]$ & $\Theta_{\text {loss }}$ \\
\hline True optimum & 1.327 & 0 \\
Open-loop nominal solution & 0.737 & 0.802 \\
Solution Model A (NCO tracking) & 1.325 & 0.002 \\
Solution Model B (NCO tracking) & 1.314 & 0.010 \\
Solution Model C (NCO tracking) & 1.223 & 0.085 \\
\hline
\end{tabular}

\section{Robustness of the Solution Models}

Here, the solution models developed for the tendency process model are applied to a detailed process model. The detailed process model is an equilibrium stage model and represents the binary distillation of a cyclohexane - n-heptane mixture in a packed column. ${ }^{23}$ This model assumes:

- negligible vapor holdup,

- perfect mixing,

- total condenser without sub-cooling.

The liquid temperatures are determined from a dynamic energy balance on each stage. Stage and condenser liquid holdups are modeled through weir equations, which also determine liquid flow rates. Vapor flow rates depend on pressure drop and thus differ from stage to stage. Physical properties, which are assumed to be composition- and temperature-dependent, are used in the energy balance, liquid holdup and the pressure drop calculations. This process model, referred to as the detailed process model, contains $3(p+1)$ differential equations, where $p=20$ is the number of stages including the reboiler. Hence, the detailed column model is of $63^{\text {rd }}$ order, while the tendency process model introduced earlier is of $3^{\text {rd }}$ order.

\section{Problem Formulation}

As for the tendency process model, we restrict our attention to a problem in which the final time $t_{f}$ is specified (e.g., the time available in one operating shift). The objective is to maximize the amount of distillate $J$ while meeting a purity constraint on the accumulated distillate composition $x_{D}$. The more volatile cyclohexane is the primary component of the distillate. The manipulated variable is the internal reflux ratio $r(t)$, which is constrained between no reflux, $r=0$, and total reflux, $r=1$.

Increasing the reflux ratio improves the distillate purity but reduces its production rate. Thus, the optimal reflux profile will seek a compromise between quantity and quality. Conceptually, 
the reboiler duty and the column pressure are additional manipulated variables. However, maximizing production requires operation at maximum pressure drop, ${ }^{16}$ which determines the reboiler duty. Furthermore, the separation of thermally degradable components calls for an upper limit on temperature, which fixes the maximal pressure. Without this limit, economic considerations would suggest operating the column at maximal pressure. Hence, the reboiler duty and the column pressure are not considered as manipulated variables here, but they are fixed at upper bounds related to maximal pressure drop and economic considerations (column design), respectively.

The optimization problem can be expressed mathematically as follows:

$$
\begin{aligned}
\max _{r(t)} J= & \sum_{c=1}^{n} \sum_{i=1}^{p+1} H_{i, c}(0)-H_{i, c}\left(t_{f}\right) \\
\text { s.t. } \quad & \text { dynamic process model } \\
& 0 \leq r(t) \leq 1 \\
& x_{D}\left(t_{f}\right) \geq x_{D, \text { des }}
\end{aligned}
$$

where $H_{i, c}$ is the molar holdup of component $c, c=1, \ldots, n$, on stage $i, i=1, \ldots,(p+1)$. The total number of components is $n$ (here $n=2$ ), $p$ is the number of stages including the reboiler $(i=1)$ and the condenser $(i=p+1)$. The amount of distillate at final time is expressed as the difference between the initial and final total holdups. The final time is fixed at $t_{f}=3 h$, and the desired final distillate composition is $x_{D \text {,des }}=0.95 \mathrm{kmol} / \mathrm{kmol}$.

\section{Loss Function for Detailed Process Model}

Next, the Solution Models A-C generated using the tendency process model are used to optimize the more detailed process model. The results are listed in Table 4. Though the cost values are different from those in Table 3, the loss functions are similar. This indicates that the solution models exhibit about the same amount of approximation capability for both the tendency and the detailed process models. Hence, the solution models are rather robust with respect to plant/model mismatch and it seems reasonable to want to apply Solution Models A or B to the real plant.

Table 4: Cost $J$ and loss function $\Theta_{\text {loss }}$ for the detailed process model. The solution models A-C are generated from numerical optimization of the tendency process model.

\begin{tabular}{ccc}
\hline Strategy & $J[\mathrm{kmol}]$ & $\Theta_{\text {loss }}$ \\
\hline True optimum & 1.399 & 0 \\
Open-loop nominal solution & 0.777 & 0.800 \\
Solution Model A (NCO tracking) & 1.398 & 0.001 \\
Solution Model B (NCO tracking) & 1.374 & 0.018 \\
Solution Model C (NCO tracking) & 1.304 & 0.073 \\
\hline
\end{tabular}

\section{Verification over a Range of Conditions}

Last section has proposed a procedure to validate a solution model for optimizing a real plant. Step 2 concerned with the assessment of model mismatch between the plant model and the real 
plant is usually the most difficult one. This step can be replaced by evaluating the proposed solution models over a range of parameter values or operating conditions. In the following, the difficulty of separation and the condenser holdup are varied in the tendency process model.

\section{Difficulty of Separation}

The difficulty of separation is investigated by varying the relative volatility in the range $1.3 \leq \alpha \leq 2.2$. Using Solution Model A, the optimal values of the input parameters $t_{1}, t_{2}, r_{1}$ and $r_{2}$ and the cost function $J$ are given in Figures 3 and 4 . For a difficult separation (low relative volatility), the reflux ratio is high, as expected. Also, the third interval disappears $\left(t_{2}=t_{f}\right)$ since the condenser composition is no longer higher than the desired distillate composition. Note that operation becomes infeasible for $\alpha<1.25$ as the distillate composition never reaches the desired composition at full reflux.
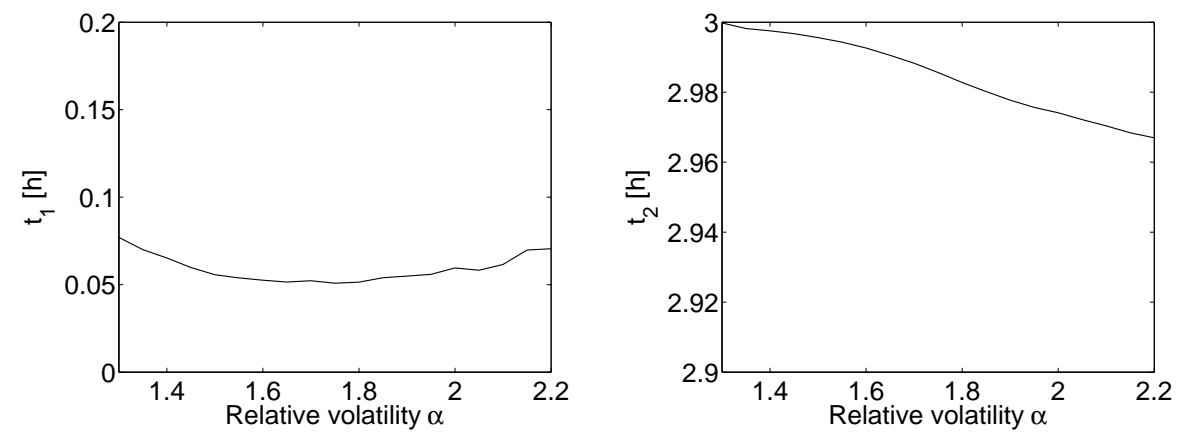

Figure 3: Optimal switching times $t_{1}$ and $t_{2}$ for Solution Model A over a range of relative volatilities.
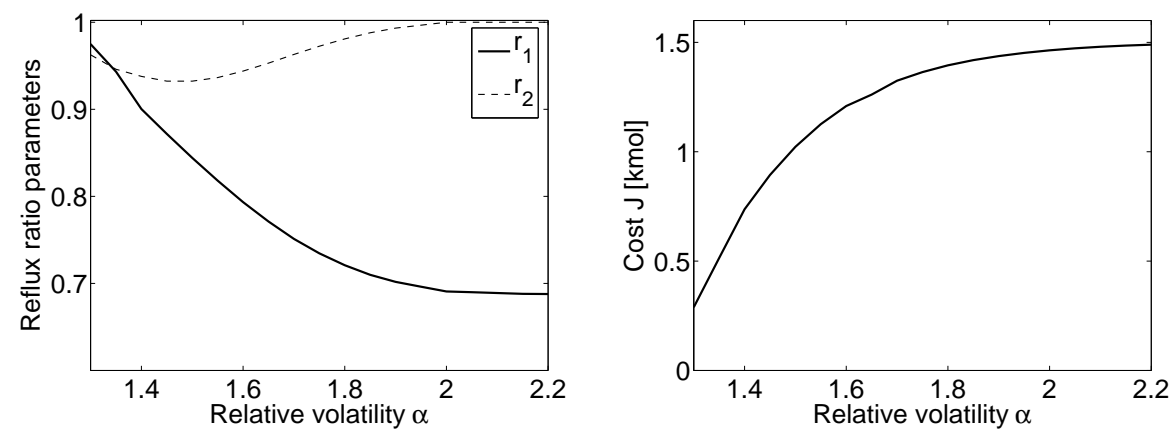

Figure 4: Reflux ratio parameters $r_{1}$ and $r_{2}$ for Solution Model A, and corresponding cost function $J$, over a range of relative volatilities.

On the other hand, for an easy separation, the length of Interval c increases. Note that the reflux ratio $r_{1}$ (value at beginning of Interval b) decreases with increasing $\alpha$, while $r_{2}$ (final value in Interval $\mathrm{b}$ ) increases with $\alpha$ for $\alpha>1.5$. This can be explained by the need to withdraw all the light component from the reboiler, which requires the reflux ratio to be set to full reflux at the end of Interval b. Also, the fixed time of operation allows increasing $r_{2}$ for high relative volatilities since $r_{1}$ is decreased.

Figure 5 shows the loss function $\Theta_{\text {loss }}$ computed as follows: The tendency model with relative volatility $\alpha$ serves as the plant, and NCO tracking is implemented with Solution Models A, B and C. It is seen that Solution Model A is perfectly valid for all values of $\alpha$ since $\Theta_{\text {loss }}$ is nearly zero everywhere. In contrast, $\Theta_{\text {loss }}$ increases with $\alpha$ for Solution Model B, and it is significantly larger for Solution Model C.

\section{Stage and Condenser Holdups}




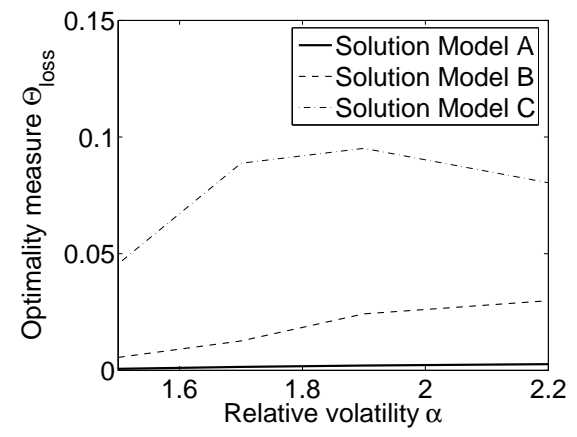

Figure 5: Loss function $\Theta_{\text {loss }}$ for various solution models over a range of relative volatilities.

Since the length of Intervals a and c in Solution Model A are linked to the stage and condenser holdups, the holdups represent an important feature of the model. The initial interval is necessary to accumulate the light component in the head of the column, while Interval $\mathrm{c}$ is utilized to recover the high-quality product that is still present in the condenser at the end of Interval b. The tendency process model does not incorporate the stage holdups explicitly, but lumps them together with the condenser holdup. Therefore, setting the condenser holdup to zero will eliminate Intervals a and $\mathrm{c}$ in the optimal solution, i.e. $t_{1}=0$ and $t_{2}=t_{f}$ as shown in Figure 6. Here, the condenser holdup $H_{p+1}$ is varied from 0 to $1 \mathrm{kmol}$, while holding the reboiler holdup constant. As expected, increasing the condenser holdup lengthen the startup phase in the optimal solution.

Figure 7 shows the reflux parameters $r_{1}$ and $r_{2}$ and the loss function $\Theta_{\text {loss }}$ computed as follows: The nominal tendency model with condenser holdup $H_{p+1}$ serves as the plant, and NCO tracking is implemented with Solution Models A, B and C. The measure $\Theta_{\text {loss }}$ is nearly zero with Solution Model A but increases with increasing condenser holdup with Solution Models B and C. Hence, Solution Model A that includes Interval c is valid for all values of $H_{p+1}$, which is not the case for Solution Models $\mathrm{B}$ and $\mathrm{C}$ that do not include Interval c.
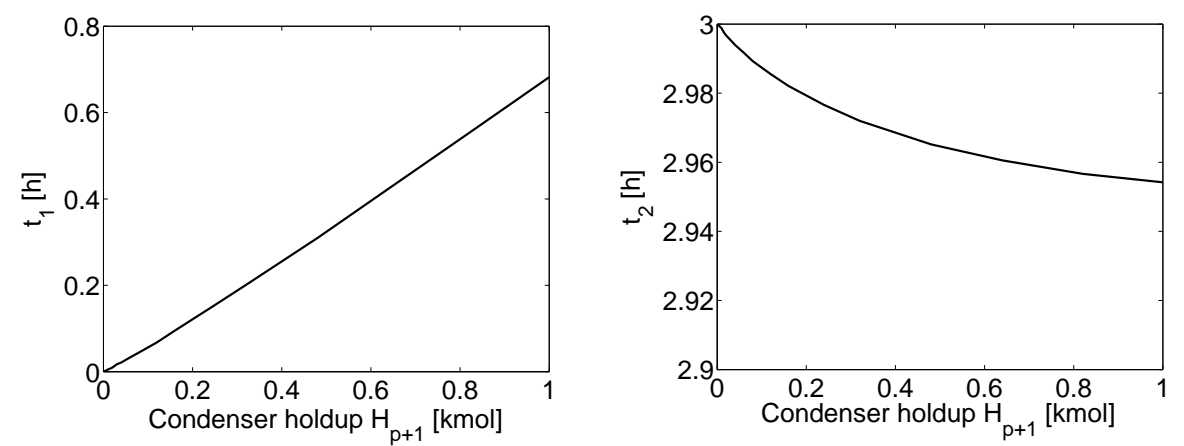

Figure 6: Optimal switching times $t_{1}$ and $t_{2}$ for Solution Model A over a range of condenser holdups.

\section{Conclusions}

This paper has proposed a loss function for validating the input parameterization in the context of NCO tracking for dynamic optimization problems. Using this loss function, the applicability of a given solution model to different process models or uncertainty realizations can be assessed. A procedure has been proposed to validate the applicability of a solution model to a real plant. 

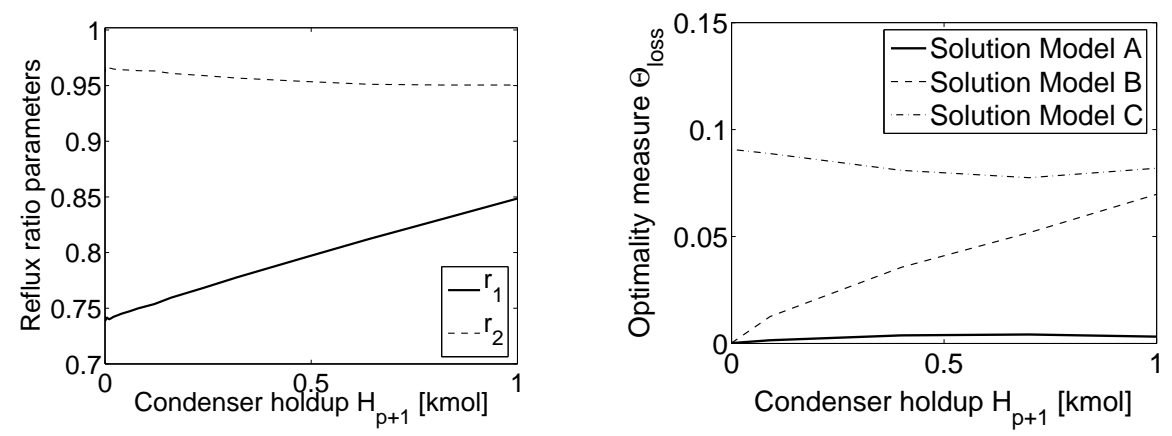

Figure 7: Reflux ratio parameters $r_{1}$ and $r_{2}$ for Solution Model A, and loss function $\Theta_{\text {loss }}$, over a range of condenser holdups.

The focus in this paper has been on comparing different solution models for optimizing two process models of widely different complexity developed for a binary batch distillation column. It has been shown that a solution model developed from a $3^{r d}$-order process model is applicable with nearly equal performance to a $63^{r d}$-order process model, thereby verifying the conjecture that the solution model derived from a simple process model is appropriate to optimize a more detailed process model. The results presented are promising but need to be supported by additional studies, in particular an application to a real process. Furthermore, it would be of interest to investigate the validity of solution models in the presence of other types of uncertainty such as process disturbances.

\section{References}

1. Srinivasan B, Bonvin D. Dynamic optimization under uncertainty via NCO tracking: A solution model approach. In: BatchPro Symposium: Poros, Greece: 2004:17-35.

2. Srinivasan B, Bonvin D, Visser E, Palanki S. Dynamic optimization of batch processes: II. Role of measurements in handling uncertainty. Comp Chem Eng. 2003;27:27-44.

3. Bryson AE, Ho YC. Applied Optimal Control. Washington DC: Hemisphere Publishing Corporation, 1975.

4. Sirisena HR. Computation of optimal controls using a piecewise polynomial parameterization. IEEE Trans Autom Contr. 1973;18:409-411.

5. Fernholz G, Engell S, Kreul LU, Gorak A. Optimal operation of a semi-batch reactive distillation column. Comp Chem Eng. 2000;24:1569-1575.

6. Schlegel M, Marquardt W. Detection and exploitation of the control switching structure in the solution of dynamic optimization problems. J Process Contr. 2006;16:275-290.

7. Hale ET, Qin SJ. Multi-parametric nonlinear programming and the evaluation of implicit optimization model adequacy. In: DYCOPS \%: Cambridge, USA: 2004.

8. Cuthrell JE, Biegler LT. Simultaneous optimization and solution methods for batch reactor control profiles. Comp Chem Eng. 1989;13:49-62.

9. Diwekar UM. Batch Distillation: Simulation, Optimal Design and Control. Washington DC: Taylor \& Francis, 1995. 
10. Mujtaba IM, Macchietto S. Optimal operation of multicomponent batch distillation - Multiperiod formulation and solution. Comp Chem Eng. 1993;17:1191-1207.

11. Robinson ER. The optimization of batch distillation operations. Chem Eng Science. 1969;24:1661-1668.

12. Hansen TH, Jorgensen SB. Optimal Control of Binary Batch Distillation in Tray or Packed Columns. Chem Eng J. 1986;33:151-155.

13. Diwekar UM, Malik RK, Madhavan KP. Optimal reflux rate policy determination for multicomponent batch distillation columns. Comp Chem Eng. 1987;11:629-637.

14. Farhat S, Pibouleau L, Domenech S, Czernicki M. Optimal Control of Batch Distillation via Nonlinear Programming. Chem Eng Process. 1991;29:33-38.

15. Kim YH. Optimal design and operation of a multi-product batch distillation column using dynamic model. Chem Eng Process. 1999;38:61-72.

16. Li P, Wozny G. Tracking the predefined optimal policies for multiple-fraction batch distillation by using adaptive control. Comp Chem Eng. 2001;25:97-107.

17. Egly H, Ruby V, Seid B. Optimum design and operation of batch rectification accompanied by chemical reaction. Comp Chem Eng. 1979;3:169-174.

18. Cuille PE, Reklaitis GV. Dynamic simulation of multicomponent batch rectification with chemical reactions. Comp Chem Eng. 1986;10:389-398.

19. Sorensen E, Macchietto S, Stuart G, Skogestad S. Optimal control and on-line operation of reactive batch distillation. Comp Chem Eng. 1996;20:1491-1498.

20. Shinskey FG. Process Control Systems. New York: McGraw-Hill, 2nd ed. 1979.

21. Srinivasan B, Palanki S, Bonvin D. Dynamic optimization of batch processes: I. Characterization of the nominal solution. Comp Chem Eng. 2003;27:1-26.

22. Hocking LM. Optimal Control: An Introduction to the Theory with Applications. New-York: Oxford University Press, 1991.

23. Welz C, Srinivasan B, Bonvin D, Ricker NL. Modeling of batch distillation columns. Technical Report, Laboratoire d'Automatique, École Polytechnique Fédérale de Lausanne, CH-1015 Lausanne, Switzerland 2005. 